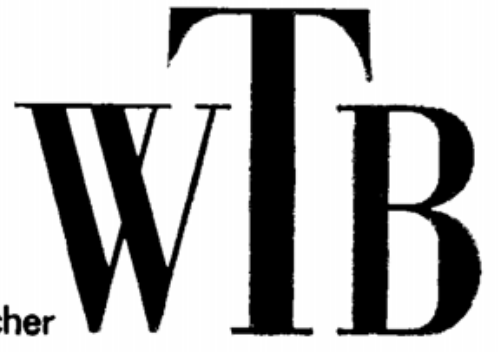

\title{
Dieter Onken
}

\section{Chemie}

Akademie-Verlag · Berlin

Pergamon Press · Oxford

Vieweg + Sohn - Braunschweig 
Wissenschaftliche Taschenbücher

ROLF BORSDORF / MANFRED SCHOLZ

Spektroskopische Methoden in der organischen Chemie

WERNER HABERDITZI

Magnetochemie

GERHARD HEBER

Mathematische Hilfsmittel der Physik, Teil I und II

A. A. SOKOLOW

Elementarteilehen

HEINZ AHRENS

Varianzanalyse

HANS-J URGEN TREDER

Relativität und Kosmos

Raum und Zeit in der Physik, Astronomie und Kosmologie

ALBERT EINSTEIN

Grundzüge der Relativitätstheorie

ALBERT EINSTEIN

Ùber die spezielle und die allgemeine Relativitätstheorie

G UNTHER LUDWIG

Wellenmechanik. Einführung und Originaltexte

HARRY PAUJ.

Lasertheorie, Teil I und II

FRANZ RUDOLF KESSLER

Einführung in die physikalischen Grundlagen

der Kernenergiegewinnung

EBERHARD HOFMAN N

Eiweiße und Nucleinsäuren als biologische Makromoleküle

Dynamische Biochemie, 'Teil I

EBERHARD TEUSCHER

Pharmakognosie, Teil I und Il 
D. TER HAAR

Quantentheorie. Einführung und Originaltexte

J. H. SAN D E R S

Die Lichtgeschwindigkeit. Einführung und Originaltexte

JEAN KUNTZMANN

Unendliche Reihen

Mathematische Hilfsmittel der Physik und Chemie

Mit 94 Ủbungen und 29 Aufgaben

JEAN KUNTZMAN N

Systeme von Differentialgleichungen

Mathematische Hilfsmittel der Physik und Chemie

Mit 88 U̇bungen und 40 Aufgaben

JEAN KUNTZMANN

Komplexe Veränderliche

Mathematische Hilfsmittel der Physik und Chemie

Mit 90 Ưbungen und 37 Aufgaben

FER DINAND CAP

Einführung in die Plasmaphysik

I. Theoretische Grundlagen

- insgesamt 3 Bände -

J. A. ROSANOW

Wahrscheinlichkeitstheorie

HAR RY PFEIFER

Theorie linearer Bauelemente

Elektronik für den Physiker I

HARRY PFEIFER

Die Elektronenröhre

Elektronik für den Physiker II

HARRY PFEIFER

Schaltungen mit Elektronenröhren

Elektronik für den Physiker III

SIEGFRIED HAUPTMANN

Utber den Ablauf organisch-chemischer Reaktionen 
GERHARD HÜBNER / KLAUS JUNG / ECKART WINKLER Die Rolle des Wassers in biologischen Systemen

STEPHEN G. BRUSH

Kinetische Theorie, Teil I und II

HARRY PFEIFER

Leitungen und Antennen

Elektronik für den Physiker IV

HAR RY PFEIFER

Mikrowellenelektronik

Elektronik für den Physiker V

HAR RY PFEIFER

Halbleiterphysik

Elektronik für den Physiker VI

EBERHARD HOFMAN N

Enzyme und energieliefernde Stoffwechselreaktionen

Dynamische Biochemie, Teil II

PETER KRUMBIEGEL

Isotopieeffekte

D. M. BRIN K

Kernkrääfte. Einführung und Originaltexte

HERBERT GO ERING

Elementare Methoden

zur Lösung von Differentialgleichungsproblemen

DIETER ONKEN

Stereoide

Zur Chemie und Anwendung 